\title{
Broadband Gain-Spectrum Measurement for Fiber Optical Parametric and Raman Amplifiers
}

\author{
V. Gordienko, M. F. C. Stephens, and N. J. Doran
}

\begin{abstract}
We examine the use of a depolarized broadband probe to experimentally measure gain spectra of amplifiers comprising parametric and Raman gain. The suggested technique allows a quick and accurate characterization of gain spectra spanning more than $100 \mathrm{~nm}$. We derive formulas for processing spectral data to address polarization dependent gain and idler generation, and consequently develop a measurement methodology for obtaining reliable results. We demonstrate the viability of this approach by performing an experimental comparison with results obtained using tunable lasers. We expect the technique described here to be useful for fiber optical parametric amplifier development and characterization.
\end{abstract}

Index Terms-Gain measurement, Optical parametric amplifiers, Raman scattering, Broadband amplifiers.

\section{INTRODUCTION}

$\mathrm{G}$ ain bandwidth over $100 \mathrm{~nm}$ has been reported for a number of developing fiber optical amplification technologies such as Raman, parametric and a variety of doped fiber amplifiers (e.g. Thulium, Holmium, Bismuth, etc.) [1]-[5]. From these reports it is clear that gain spectrum characterization over such a wide bandwidth using a comb of fixed lasers or a tunable laser is difficult due to the availability of sources and/or measurement time. This is exacerbated if the amplifier possesses polarization dependent gain requiring probe polarization control.

Among the technologies discussed above, fiber optical parametric amplifiers (FOPA) represent particular interest due to the theoretically unlimited gain bandwidth achievable at arbitrary wavelengths [6]. However, the FOPA has unique features which can skew the raw measurement results obtained with a broadband (BB) probe such as idler generation [6] and significant four-wave mixing (FWM) between frequency components of the probe [7]. Additionally, FOPA polarization-dependent gain needs to be accounted for when a BB probe is used (see Section III-C for details). The first work experimentally investigating the effect of these issues in the

Manuscript received xxxxx, 2017; revised xxxxx, 2017; accepted xxxxx, 2017. Date of publication xxxxx, 2017; date of current version xxxxx, 2017. This work was funded by EPSRC project UPON, EP/M005283/1. The work of V. Gordienko was supported by II-VI.

V. Gordienko, M. F. C. Stephens, and N. J. Doran are with Aston Institute of Photonic Technologies, Aston University, Birmingham, UK (email: gordienv@aston.ac.uk, m.stephens@aston.ac.uk,n.j.doran@aston.ac.uk).

Research data are available to download at: http://doi.org/10.17036/researchdata.aston.ac.uk.00000205.

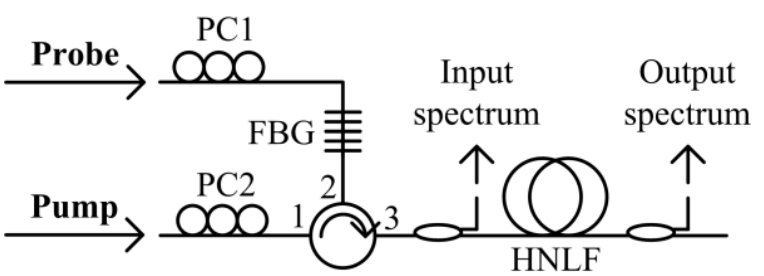

Fig. 1. Generalized FOPA experimental setup for gain spectrum characterization using a narrowband or broadband probe.

context of accurate BB FOPA characterization has just been published [8] enabling an extensive experimental study of FOPA gain spectrum [9].

In this paper we investigate both theoretically and experimentally the use of a BB probe for measuring FOPA and Raman gain spectra quickly and accurately. Raman gain is taken into consideration since it inevitably mixes with FOPA gain spanning over $100 \mathrm{~nm}$. This paper extends the previous work [8] by refining the measurement concept and thus improving measurement accuracy.

\section{EXPERIMENTAL SETUP}

The experimental setup for BB gain characterization of an amplifier comprising parametric and Raman gain is shown in Fig. 1 and is fully described in [10]. A single polarization continuous wave pump and a test probe of choice were coupled together using a stretchable fiber Bragg grating (FBG) tuned to the pump wavelength and an optical circulator [11]. The pump was phase-modulated with RF tones to mitigate stimulated Brillouin scattering [12]. The pump and a probe were co-propagated through a highly nonlinear fiber (HNLF) with zero-dispersion wavelength $\lambda_{0} \sim 1551 \mathrm{~nm}$. Tap couplers before and after the HNLF and an optical spectrum analyzer were used to record the optical spectrum at the input and output of the HNLF. Polarization controllers PC1 and PC2 were used to manipulate the probe and pump polarizations.

The BB probe for the amplifier characterization in this work was derived using a supercontinuum (SC) source [13]. The spectral shape of the probe was adjustable by tuning the SC pump power. This allowed wide bandwidth probe generation from $1520 \mathrm{~nm}$ to $1700 \mathrm{~nm}$. Importantly, the $\mathrm{SC}$ was depolarized (degree of polarization <1\%). An alternative narrowband polarized probe was also used to compare gain measurement results made with the BB probe. This was from either a $100 \mathrm{kHz}$ linewidth tunable laser for wavelengths $1550-1625 \mathrm{~nm}$ or a wavelength converted laser for 1625$1670 \mathrm{~nm}$. Further, probes are referenced using a notation X- 
$\mathrm{YY}$ where $\mathrm{X}$ is either $\mathrm{P}$ for polarized or $\mathrm{D}$ for depolarized and $\mathrm{YY}$ is either NB for narrowband or BB for broadband.

Details of the experimental setups used in Section III below are provided in the corresponding figure captions. In Section IV we then concentrate on three main configurations (A, B and C) utilizing the same $50 \mathrm{~m}$ long fiber with a fixed pump power of $5 \mathrm{~W}$. In configuration $\mathrm{A}$, the pump wavelength is set at $1565 \mathrm{~nm}$ to restrict the parametric gain to a small region near the pump and examine the parametric and Raman gain independently. In configurations $\mathrm{B}$ and $\mathrm{C}$, the pump wavelength is tuned close to $\lambda_{0}$ to obtain parametric gain spanning over $100 \mathrm{~nm}$, thus observing mixing of parametric and Raman gains provided by the same pump. In configurations $\mathrm{A}$ and $\mathrm{B}$ the pump polarization is tuned to maximize the parametric gain ensuring linearity of the pump polarization (linear pump polarization allows the highest FWM coupling efficiency [14]). In configuration C the pump polarization is tuned to suppress the parametric gain peak, so it can be stated that the pump polarization is not linear in this case.

\section{BROADBAND GAIN SPECTRUM MEASUREMENT}

Measuring a gain spectrum using a $\mathrm{BB}$ probe requires finding the difference between the input and output power spectra of the amplifier under test. The gain can be measured at every frequency where the BB probe exists. The BB probe does not need to be spectrally flat as the gain is calculated independently at each frequency. However, in the general case of an amplifier comprising both Raman and parametric amplification, the gain spectrum consists of parametric-only and Raman-only regions as well as regions with substantial Raman and parametric gain occurring together, as shown in Fig. 2. This creates several issues for determining the correct gain at all BB probe frequencies, discussed below.

\section{i. Signal-idler mixing}

Parametric gain is symmetric around a central frequency, $f_{c}$ [6]. If the BB probe straddles $f_{c}$ at the input then the output spectrum will unavoidably represent a mix of signals and idlers, where 'signals' refer to waves being amplified, and 'idlers' are copies of signals symmetric with them around $f_{c}$. Mixing of signals and idlers does not allow the gain to be calculated as a ratio between output and input powers because in this case the output power at every frequency is a sum of the amplified signal and an idler. We either need to resolve the signal-idler mixing or restrict the input $\mathrm{BB}$ probe to strictly one side of $f_{c}$ per measurement. This is difficult to achieve experimentally without a 'blind-spot' occurring around $f_{c}$, and therefore typically the BB probe will straddle $f_{c}$. The resultant signal-idler mixing can be expressed in linear units as:

$$
P_{1}^{\prime}=P_{1} \times G_{s}+P_{2} \times G_{i},
$$

where $P_{1}^{\prime}$ and $P_{1}$ are output and input signal powers at the particular frequency, $P_{2}$ is the input signal power at the symmetric frequency, $G_{s}$ is the signal gain and $G_{i}$ is the signal-to-idler conversion efficiency. Equation(1) can be solved in terms of $G_{s}$ in regions with parametric gain only by

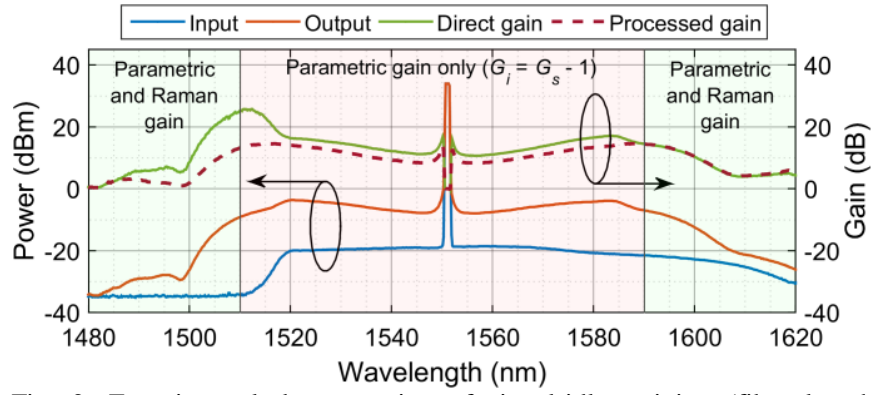

Fig. 2. Experimental demonstration of signal-idler mixing (fiber length $100 \mathrm{~m}$, pump wavelength $1551 \mathrm{~nm}$, pump power $2 \mathrm{~W}$ ). Wavelength ranges corresponding to different idler gains are highlighted. Direct gain (green curve) is found as a ratio between measured output (orange curve) and input (blue curve) spectra. Processed gain (broken red curve) is found using (2) and the same output and input spectra.

considering that $G_{s}=G_{i}+1[6]$ :

$G_{s}=\frac{P_{1}^{\prime}+P_{2}}{P_{1}+P_{2}}$.

Equation (2) can then be used as a solution to calculate the signal gain on an entire single side of $f_{c}$ as long as $P_{2}=0$ in two regions: a) where Raman affects the parametric gain and b) where Raman gain dominates. These regions are situated $\sim 5-15 \mathrm{THz}$ away from the pump [15]. In this way, signal-idler mixing is avoided in these regions and (2) shrinks to a simple ratio between output and input powers.

The input probe of Fig. 2 satisfies the above conditions as it spans the long wavelength side of $f_{c}$ plus $\sim 30 \mathrm{~nm}$ at the short wavelength side where Raman susceptibility can be neglected [16]. In this case, a gain measurement can therefore be performed in the range $1520-1620 \mathrm{~nm}$. It can be seen that calculating the direct gain as a ratio between input and output overestimates the gain derived from (2) by $\sim 3 \mathrm{~dB}$ at 1520 $1590 \mathrm{~nm}$ due to the idler generation. When there is no probe at symmetric frequencies (i.e. wavelength $>1590 \mathrm{~nm}$ ), there is no signal-idler mixing and (2) provides the same result as a direct calculation. In the absence of an input probe, at wavelengths shorter than $1515 \mathrm{~nm}$, the signal gain can be estimated according to (2) based on signal-to-idler conversion without consideration of the Raman contribution as shown in Fig. 2.

\section{ii. Signal-signal interaction and pump depletion}

Both FWM and Raman scattering can produce products of interaction between different probe frequencies, which are indistinguishable from the amplified probe and therefore their effect on measured gain cannot be compensated by numerical processing of the results. However, the power of these products scales with signal power, so their insignificance can be ensured by reducing the input probe power until the gain is independent of it. Reducing the input power also ensures the absence of pump depletion. Fig. 3 shows experimental gain spectra obtained as the BB probe input power $(\sim 10 \mathrm{dBm})$ was attenuated by different amounts up to $18 \mathrm{~dB}$. It can be seen that gain distortion around the pump caused by unwanted FWM products gets suppressed as the attenuation increases up to $15 \mathrm{~dB}$. At the same time, the gain peak increases until the attenuation reaches $15 \mathrm{~dB}$ which indicates mitigation of pump depletion. Gain spectra obtained with attenuation of $15 \mathrm{~dB}$ and 


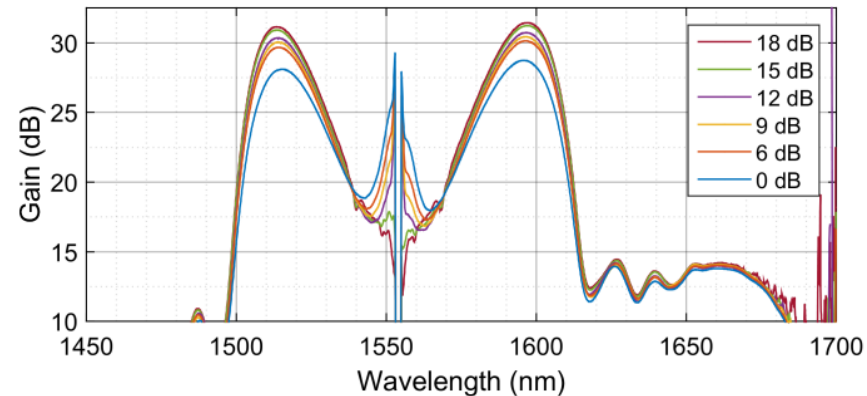

Fig. 3. Experimental gain spectra obtained for a variable attenuation of a broadband probe (fiber length $75 \mathrm{~m}$, pump wavelength $1554 \mathrm{~nm}$, pump power, $5 \mathrm{~W}$ ). Attenuation increase up to $15 \mathrm{~dB}$ completely suppresses unwanted FWM products and mitigates pump depletion.

$18 \mathrm{~dB}$ converge indicating insignificance of remaining unwanted products and pump depletion.

\section{iii. Polarization dependent gain}

Normally, when gain of a polarization sensitive amplifier is measured with a $\mathrm{P}-\mathrm{NB}$ probe or a modulated signal, the polarization is tuned to maximize gain. A P-BB probe is not suitable for a direct maximum gain measurement as illustrated in Fig. 4 which shows input and output optical spectra of a $\mathrm{P}-\mathrm{BB}$ probe obtained by passing the $\mathrm{D}-\mathrm{BB}$ probe through a polarizer. There is a significant ripple seen on the output spectrum because different frequencies possess different polarizations due to frequency dependent polarization evolution in the HNLF, polarization controllers, etc. [16]. Therefore, gain measurement using a P-BB probe does not allow a BB gain measurement since measured gain can be interpreted only at the frequency where output power was monitored during polarization tuning. Consequently, it is necessary to use a D-BB probe to perform BB gain measurements. However, D-BB probe obtains gain averaged across all polarization states which is usually not an interest of amplifier characterization, so it is required to derive relations between $\mathrm{D}-\mathrm{BB}$ gain and $\mathrm{P}-\mathrm{NB}$ gain.

\section{iv. DEPOLARIZED PROBE FOR GAIN SPECTRUM MEASUREMENT}

Fig. 5 shows comparison of experimental results obtained

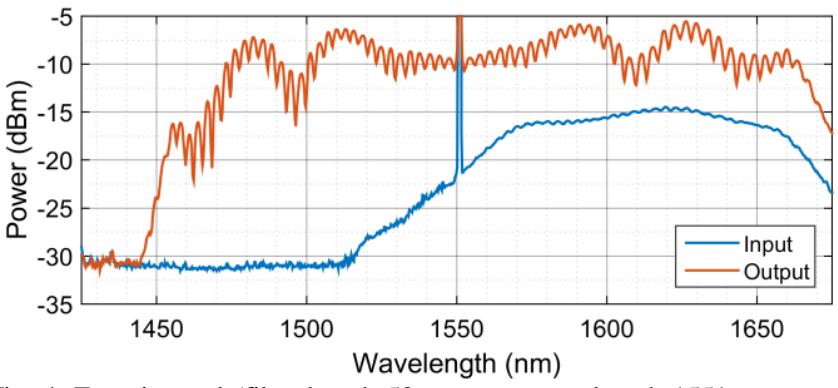

Fig. 4. Experimental (fiber length $50 \mathrm{~m}$, pump wavelength $1551 \mathrm{~nm}$, pump power $5 \mathrm{~W}$ ) input and output spectra of a polarized broadband probe demonstrate its unsuitability for a broadband gain measurement. This paper suggests to use a depolarized broadband probe instead.

with D-BB and P-NB probes for amplifier configurations A, B and $\mathrm{C}$ described in Section II. Experimentally measured D-BB gain processed using (2) is denoted as $G_{D-B B}$ (orange curve). $G_{P-N B, \max }$ (red crosses) and $G_{P-N B, \min }$ (purple crosses) are respective maximum and minimum gains of the P-NB probe measured as the polarization alignment between the P-NB probe and the pump was adjusted through the $\mathrm{P}-\mathrm{NB}$ full range at each examined frequency. The average P-NB gain $\left\langle G_{P-N B}\right\rangle$ (cyan diamonds) is calculated and plotted in Fig. 5 according to $\left\langle G_{P-N B}\right\rangle=\left(G_{P-N B \text {, max }}+G_{P-N B \text {, min }}\right) / 2$. The average P-NB gain $\left\langle G_{P-N B}\right\rangle$ corresponds to the total gain which two polarization multiplexed signals with gains $G_{P-N B \text {, max }}$ and $G_{P-N B \text {,min }}$ would receive in the examined amplifier.

A very good match between $\left\langle G_{P-N B}\right\rangle$ and $G_{D-B B}$ shows that considerations made in Sections II-A and II-B were sufficient to ensure accurate gain measurement for both parametric and Raman gain regions. Therefore, D-BB probe measurement can be used to find the total gain of polarization multiplexed signals over wide bandwidth quickly and accurately. This is usually the main interest of polarization-diverse amplifier characterization.

The agreement between $\left\langle G_{P-N B}\right\rangle$ and $G_{D-B B}$ provides a relation linking the D-BB probe gain $G_{D-B B}$ with the maximum P-NB probe gain $G_{P-N B \text {, max }}$, which is usually the main interest of polarization-sensitive amplifier characterization:

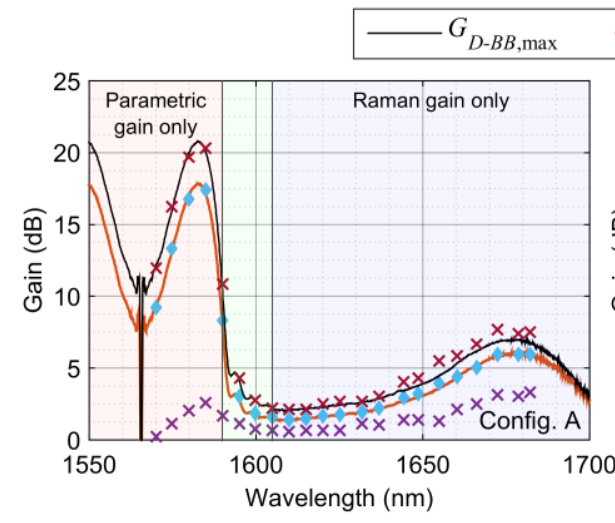

(a)

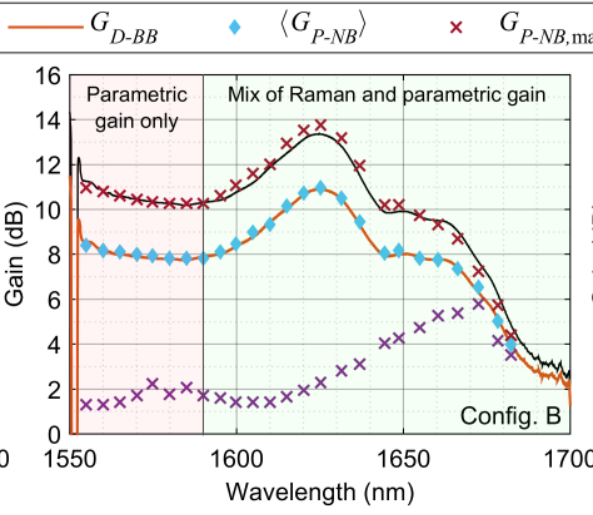

(b)

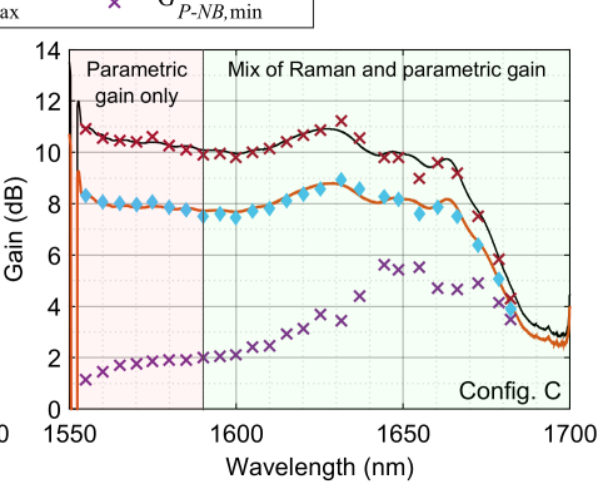

(c)

Fig. 5. Experimental results of gain spectra measurement with D-BB and P-NB probes for configurations (config.) A, B and C respectively of an examined amplifier comprising Raman and parametric gain. $G_{D-B B}$ (orange curve) is a total gain of a D-BB probe processed using (2); $G_{D-B B, \max }$ (black curve) is a maximum P-NB gain calculated as a solution of (4) and based on results of D-BB gain measurement. $G_{P-N B, \max }$ (red crosses) and $G_{P-N B, \min }$ (purple crosses) are the maximum and the minimum P-NB gain obtained by tuning the P-NB polarization. $\left\langle G_{P-N B}\right\rangle$ (cyan diamonds) is the average of $G_{P-N B \text {,max }}$ and $G_{P-N B \text {,min }}$. 


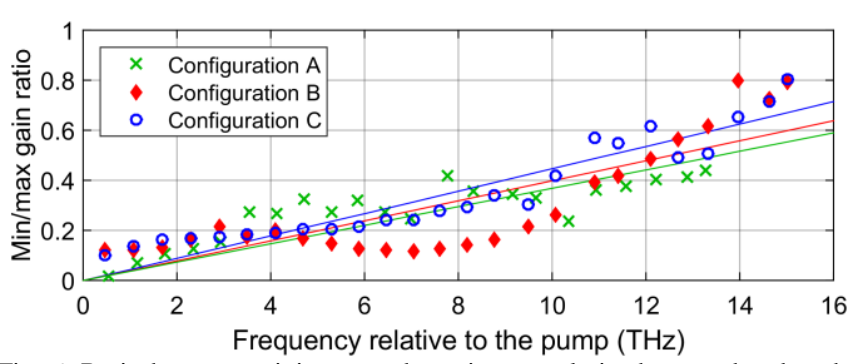

Fig. 6. Ratio between minimum and maximum polarized narrowband probe gain values represented in $\mathrm{dB}$ for three configurations of the examined amplifier. Fitted straight lines are shown with corresponding colors.

$G_{D-B B}=\left\langle G_{P-N B}\right\rangle=\frac{G_{P-N B, \max }+G_{P-N B, \min }}{2}$.

Assuming $G_{D-B B}$ to be known as a result of measurement, $G_{P-N B \text {, max }}$ still cannot be calculated using (3) unless $G_{P-N B \text {, min }}$ is known. Note, that $G_{P-N B \text {, min }}$ cannot be neglected in (3) as Fig. 5 demonstrates that $G_{P-N B \text {,min }}$ can be comparable to $G_{P-N B \text {, max }}$. One reason for an increase of notable $G_{P-N B \text {, min }}$ is the presence of Raman orthogonal susceptibility (ROS). However, the ROS peaks at $\sim 3 \mathrm{THz}(\sim 25 \mathrm{~nm})$ detuning from the pump in silica [16], so this does not explicitly explain the increase of $G_{P-N B \text {, min }}$ seen for larger P-BB probe detuning. Instead we suggest that the significant increase of $G_{P-N B \text {,min }}$ $\sim 100 \mathrm{~nm}$ away from the pump is attributed to a relative rotation of the P-NB probe and the pump polarization states due to wavelength dependent birefringence [17].

A study of the exact reasons for increase of $G_{P-N B \text {, min }}$ is beyond the scope of this paper, but it can be noticed that the ratio between the minimum and the maximum $\mathrm{P}-\mathrm{NB}$ gain $K=G_{P-N B \text {, min }}^{d B} / G_{P-N B \text {, max }}^{d B}$ (gains are expressed in $\mathrm{dB}$, and the untiless $K$ defined in this way approximates a ratio between gain coefficients) increases nearly linearly with the P-NB probe detuning from the pump (Fig. 6). The slope of fitted straight lines for all examined amplifier configurations is also very close. We suggest therefore that the maximum and minimum gain is measured with a $\mathrm{P}-\mathrm{NB}$ probe at a few points of one gain spectrum to find a ratio between them and fit it with a straight line. The fitted line then can be used to find an approximate value of $K$ for all frequencies and amplifier configurations and consequently to substitute $G_{P-N B \text {, min }}$ with $\left(G_{P-N B, \max }\right)^{K}$ in (3):

$2 G_{D-B B}=G_{P-N B, \max }+\left(G_{P-N B, \max }\right)^{K}$.

Equation (4) can be solved in terms of $G_{P-N B \text {, max }}$ numerically, where $G_{D-B B}$ is calculated using (2) based on measured input and output optical spectra of a D-BB probe. In this paper we use values of $K$ approximated by fitted lines (Fig. 6) to solve (4). The estimation for $G_{P-N B \text {, max }}$ based on the gain measurement using D-BB probe is shown in Fig. 5 as $G_{D-B B \text {, max }}$ (black curve). It demonstrates a good agreement with $G_{P-N B \text {, max }}$ across all configurations proving that the described approach relying on D-BB measurement and a few P-NB measurements allows the maximum P-NB gain $G_{P-N B \text {,max }}$ to be found over a wide bandwidth and various amplifier configurations with little error. Note, that using the actual measured values for $K$ would provide an accurate match between $G_{D-B B \text {, max }}$ and $G_{P-N B \text {, max }}$ limited by mismatch between $G_{D-B B}$ and $\left\langle G_{P-N B}\right\rangle$ only, but it is not practical for amplifier characterization as it requires a direct $G_{P-N B \text {, max }}$ measurement across the entire gain spectrum.

A rough estimation of $G_{P-N B \text { max }}$ can also be made without P-NB probe measurements at all. As follows from definition of $K$, it may take values from 0 to 1 , so solution of (4) for $G_{P-N B \text {, max }}$ lies in a range from $G_{D-B B}$ to $2 G_{D-B B}-1$ in all cases. This restricts $G_{P-N B \text {, max }}$ within a band which is less than $3 \mathrm{~dB}$ wide, thus allowing a rough estimation of $G_{P-N B \text {, max }}$ with an error below $1.5 \mathrm{~dB}$.

\section{ACKNOWLEDGMENT}

The authors wish to thank Dr Shigehiro Takasaka of Furukawa Electric Co Ltd and Dr Ian McClean of II-IV for useful discussions. Dr Atalla El-Taher of Aston University is thanked for the supercontinuum source provision.

\section{REFERENCES}

[1] Y. Emori and S. Namiki, "100 nm bandwidth flat gain Raman amplifiers pumped and gain-equalized by 12-wavelength-channel WDM high power laser diodes," in Optical Fiber Commun. Conf., San Diego, CA, 1999. DOI: 10.1109/OFC.1999.766052

[2] M. Jamshidifar, A. Vedadi, M. E. Marhic, "Continuous-wave one-pump fiber optical parametric amplifier with $270 \mathrm{~nm}$ gain bandwidth," in European Conf. on Optical Commun., Vienna, Austria, 2009, Paper 1.1.4.

[3] J. Wang et al., "Broadband silica-based thulium doped fiber amplifier employing multi-wavelength pumping," Optics Express, vol. 24, no. 20, pp. 23001-23008, Oct 2016.

[4] S. A. Fialtova et al., "Gain spectrum of the Ho-doped fiber Amplifier," Laser Phys. Lett., vol. 12, no. 9, article id 095105, Sep 2015.

[5] S. V. Firstov, "A 23-dB bismuth-doped optical fiber amplifier for a 1700-nm band," Scientific Reports, June 2016. DOI: 10.1038/srep28939

[6] M. E. Marhic et al., "Broadband fiber optical parametric amplifiers," Optics Lett., vol. 21, no. 8, pp. 573-575, Apr 1996.

[7] J. L. Blows, "Design strategy for controlling four-wave mixing-induced crosstalk between channels in a fibre optical parametric amplifier," Optics Commun., vol. 236, no. 1-3, pp. 115-122, June 2004.

[8] V. Gordienko et al., "Novel broadband gain-spectrum measurement technique for Raman and parametric amplifiers," in Optical Fiber Commun. Conf., Los Angeles, CA, 2017. Paper W2A.11.

[9] V. Gordienko et al., "Demonstration of an ultra-flat Raman-enhanced fibre optical parametric amplifier (FOPA) with $>110 \mathrm{~nm}$ gainbandwidth," in 42nd European Conf. on Optical Commun., Dusseldorf, Germany, 2016, pp. 1019-1021.

[10] V. Gordienko et al., "Ultra-flat wideband single-pump Raman-enhanced parametric amplification," Optics Express, vol. 25, no. 5, pp. 48104818, Mar 2017.

[11] M. F. C. Stephens et al., "The impact of pump phase-modulation and filtering on WDM signals in a fibre optical parametric amplifier," in Optical Fiber Commun. Conf., Los Angeles, CA, 2015. DOI: 10.1364/OFC.2015.W2A.43

[12] J. B. Coles et al., "Bandwidth-efficient phase modulation techniques for stimulated Brillouin scattering suppression in fiber optic parametric amplifiers," Optics Express, vol. 18, no. 17, pp. 18138-18150, Aug 2010.

[13] A. E. El-Taher et al., "High efficiency supercontinuum generation using ultra-long Raman fiber cavities," Optics Express, vol. 17, no. 20, pp. 17909-17915, Sep 2009.

[14] M. E. Marhic, "Vector OPA theory," in Fiber Optical Parametric Amplifiers, Oscillators and Related Devices, New York: Cambridge Univ. Press, 2008, ch. 4, sec. 2.3, pp. 82-84.

[15] A. S. Y. Hsieh et al., "Combined effect of Raman and parametric gain on single-pump parametric amplifiers," Optics Express, vol. 15, no. 13, pp. 8104-8114, June 2007. 
[16] Q. Lin and G. P. Agrawal, "Raman response function for silica fibers," Optics Lett., vol. 31, no. 21, pp. 3086-3088, Nov 2006.

[17] W. Eickhoff, Y. Yen and R. Ulrich, "Wavelength dependence of birefringence in single-mode fiber," Applied Optics, vol. 20, no. 19, pp. 3428-3435, Oct 1981. 